\title{
Serum Aminotransferase Activity as a Predictor for Estimation of Total Clearance of Hepatically Metabolized Drugs in Rats with Acute Hepatic
} Failure

\author{
Koichi Yokogawa,,${ }^{a, b}$ Akane Ido, ${ }^{a}$ Takeshi Kurihara, ${ }^{a}$ Yasunari Mano, ${ }^{a}$ Masaaki Nomura, ${ }^{a}$ \\ Junko IsHIZAKI, ${ }^{c}$ and Ken-ichi MiYAmoto, ${ }^{* a, b}$ \\ ${ }^{a}$ Department of Pharmacy and Health Science, Graduate School of Natural Science and Technology; Kakuma, Kanazawa \\ 920-1192, Japan: ${ }^{b}$ Department of Hospital Pharmacy, School of Medicine, Kanazawa University; 13-1 Takara-machi, \\ Kanazawa 920-8641, Japan: and ${ }^{c}$ Clinical Pharmacy Research Center, Division of Pharmaceutical Sciences, Graduate \\ School of Natural Science and Technology; Kakuma, Kanazawa 920-1192, Japan.
}

Received June 3, 2005; accepted September 7, 2005

The levels of serum aminotransferase activity, including aspartate aminotransferase $(A S T)$, in rats with acute hepatic failure at $24 \mathrm{~h}$ after an oral administration of $\mathrm{CCl}_{4}(0.01-0.5 \mathrm{ml} / \mathrm{kg})$ were about $15-50$ times higher (up to nearly $5000 \mathrm{IU} / \mathrm{l}$ ) than those of vehicle control rats (about $85 \mathrm{IU} / \mathrm{l}$ ). The values of total clearance $\left(C L_{\text {tot }}\right)$ of cyclosporin $\mathrm{A}$, doxorubicin, tacrolimus and zonisamide in the $\mathrm{CCl}_{4}$-treated rats were decreased to about $1 / 2-1 / 3$ of those in control rats. There were good correlations between $A S T$ activity and hepatic intrinsic clearance $\left(C L_{\text {int }}\right)(r=0.733-0.949)$ for the above drugs, as well as for chlorzoxazone, caffeine, lidocaine and tolbutamide after the intravenous administration of each drug in rats with acute hepatic failure. However, the slope of the linear regression equation, i.e., the ratio of decrease of $C L_{\text {int }}$ against increase of $A S T$ activity, differed markedly among these drugs. We found that there is a good correlation $(r=0.953)$ between the values of the slope and the $C L_{\text {int }}$ of normal rats for these drugs, except for caffeine. In summary, the linear regression equation enables us to predict the decrease of $C L_{\text {tot }}$ in rats with acute hepatic failure to be predicted from the increase in serum $A S T$ activity. This approach may be useful as a guide for the dose modification of drugs for patients with acute hepatic failure.

Key words serum aminotransferase activity; total clearance; hepatic intrinsic clearance; acute hepatic failure

In treating patients with hepatic failure, it is necessary to set an appropriate drug dosing schedule to take into account of their altered drug disposition kinetics. However, little work has been done to estimate the decrease of total clearance $\left(C L_{\text {tot }}\right)$ in relation to the degree of residual hepatic function. Therefore, there is great need for a suitable marker to quantitatively predict the hepatic metabolic capacity in patients with hepatic failure.

In humans, there are five predominant families of cytochrome P450 (CYP) species, CYP1A, CYP2C, CYP2D, CYP2E and CYP3A; all of the isoforms differ in their patterns of drug-metabolizing activity, ${ }^{1)}$ and the amounts of the isoforms in the liver are altered under conditions of hepatic failure. $^{2)}$ The drug-metabolizing activity of the liver may be evaluated on the basis of the correlation between the clearance and the ratio of the metabolite(s) to unchanged drug after the administration of specific inhibitors of various CYP isoforms.

Recently, there have been many reports on the expression levels of CYP isoform mRNAs in human liver collected by hepatobiopsy, but no clear correlation has been found between expression levels and the degree of hepatic failure. ${ }^{3,4)}$ Finnstrom et al. reported that the expression of CYP mRNA in liver is different from that in blood; therefore, blood cannot serve as a surrogate organ for assessment of the expression of the CYP genes in liver. ${ }^{5)}$ Panduro et al. ${ }^{6)}$ reported that liver regeneration and fibrosis are related to $\gamma$-carboxylase activity, which is associated with a prolongation of prothrombin time in plasma in rats treated with $\mathrm{CCl}_{4}$. Clinically, indocyanine green is commonly used to assess hepatic function. ${ }^{7)}$ However, none of these procedures appears to be suitable for predicting the decrease in hepatic metabolic activity in patients with hepatic failure.

It has been proposed that the drug-metabolizing activity of the liver can be estimated by means of pharmacokinetic analysis after the administration of various probe drugs for CYP isoforms. ${ }^{8-11)}$ Matsuo et al. demonstrated that there is a correlation between the $C L_{\text {tot }}$ of cyclosporine and the grade severity of liver disease by Child-Pugh classification in patients with hepatitis. ${ }^{12)}$ However, it is not easy to utilize these approaches to determine the degree of residual hepatic metabolizing ability in patients, as is also the case with creatinine clearance in patients with renal failure.

We previously reported that there is a good correlation between mRNA expression and enzyme activity of various CYP isoforms in the livers of rats with acute hepatic failure induced by various doses of $\mathrm{CCl}_{4}{ }^{13)}$ In that study, we also found a good negative correlation between serum aminotransferase activity $(A S T)$ and the hepatic activity of each CYP isoform, though the degree of decrease of $C L_{\text {tot }}$ varied markedly among CYP isoforms.

Here, we present a novel procedure to predict the decrease of $C L_{\text {tot }}$, including that for unknown drugs, based on an increase in serum $A S T$ activity in rats with acute hepatic failure induced by $\mathrm{CCl}_{4}$.

\section{MATERIALS AND METHODS}

Materials Adriacin injection ${ }^{\circledR}$ (doxorubicin hydrochloride) was purchased from Kyowa Hakko Kogyo Co., Ltd. (Tokyo, Japan). Caffeine, tolbutamide and $\mathrm{CCl}_{4}$ were purchased from Wako Pure Chemicals Co., Ltd. (Osaka, Japan). 
Chlorzoxazone and lidocaine hydrochloride were purchased from Sigma-Aldrich Co., Ltd. (MO, U.S.A.). Sandimmun injection $^{\circledR}$ (cyclosporin A) was purchased from Novartis Pharma, Ltd. (Tokyo, Japan). Prograf injection ${ }^{\circledR}$ (tacrolimus) was purchased from Fujisawa Pharmaceutical Co., Ltd. (Osaka, Japan). Zonisamide was supplied by Dainippon Pharmaceutical Co., Ltd. (Osaka, Japan). Diagnogreen injection $^{\circledR}$ (indocyanine green) was purchased from Daiichi Pharmaceutical Co., Ltd. (Tokyo, Japan). All other chemicals were of reagent grade.

Preparation of Acute Hepatic Failure Rats Acute hepatic failure was induced by oral administration of $\mathrm{CCl}_{4}$ to male Wistar rats (8 weeks old, Nippon SLC Co., Ltd., Hamamatsu, Japan), which were used $24 \mathrm{~h}$ after the administration. ${ }^{14,15)} \mathrm{CCl}_{4}$ was dissolved in corn oil and administered at a dose of $0.01,0.025,0.1,0.25$ or $0.5 \mathrm{ml} / \mathrm{kg}$ in a volume of $2 \mathrm{ml} / \mathrm{kg}$ to rats. The untreated control rats received corn oil alone.

Determination of Serum Aminotransferase Activity Blood samples were collected from the jugular vein of rats with acute hepatic failure under light ether anesthesia before the administration of various drugs at $24 \mathrm{~h}$ after an oral administration of $\mathrm{CCl}_{4}$. The serum was separated by centrifugation and stored at $-30{ }^{\circ} \mathrm{C}$. Measurement of $A S T$ activity was conducted by SRL Co., Ltd. (Tokyo, Japan).

Determination of Hepatic Blood Flow Hepatic blood flow $\left(Q_{\mathrm{h}}\right)$ was determined using the method of Yokota et al. ${ }^{16)}$ Each rat was cannulated in the hepatic vein. A priming dose of indocyanine green was injected into the femoral vein, followed immediately by a constant infusion of the dye into
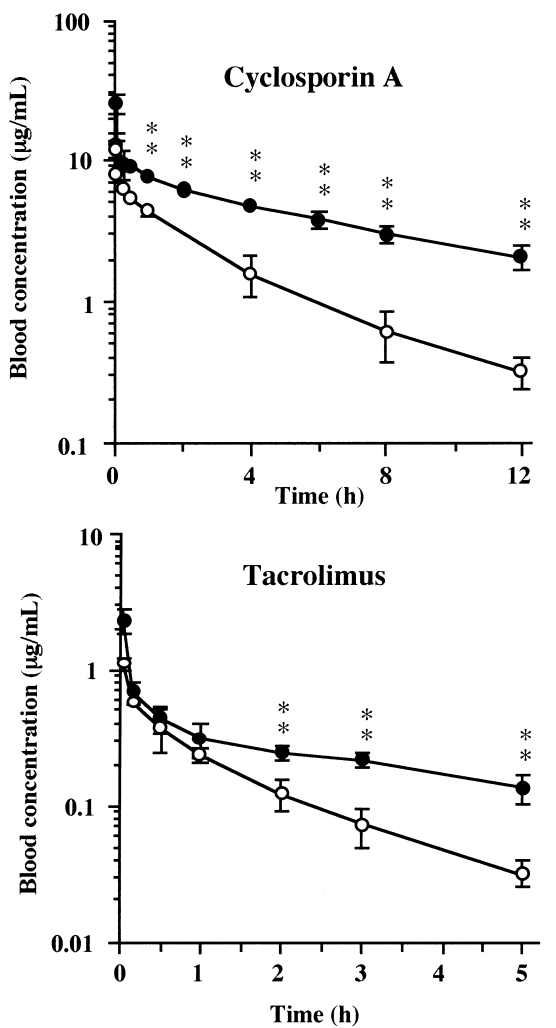

the femoral vein through the cannula at a rate of $12-13 \mu \mathrm{g} / \mathrm{min}$, yielding a steady-state plasma level of 10 $20 \mu \mathrm{g} / \mathrm{ml}$. Two blood samples (each $0.2 \mathrm{ml}$ ) were simultaneously withdrawn from the hepatic vein and femoral artery over $10 \mathrm{~min}$, starting $60 \mathrm{~min}$ after initiation of the dye injection. Plasma was separated by centrifugation. Aliquots $(50$ $100 \mathrm{ml}$ ) of plasma were diluted 10 - to 20 -fold with distilled water and quantified by measurement of the absorbance at $800 \mathrm{~nm}$

Animal Experiments A $100 \mu \mathrm{l}$ aliquot of caffeine $(5 \mathrm{mg} / \mathrm{kg})$, cyclosporin A (10 mg/kg), doxorubicin $(5 \mathrm{mg} / \mathrm{kg})$, lidocaine $(10 \mathrm{mg} / \mathrm{kg})$, tacrolimus $(2 \mathrm{mg} / \mathrm{kg})$ or tolbutamide $(10 \mathrm{mg} / \mathrm{kg})$ in distilled water, or chlorzoxazone $(10 \mathrm{mg} / \mathrm{kg})$ in $0.1 \mathrm{~N} \mathrm{NaOH}$, or zonisamide $(10 \mathrm{mg} / \mathrm{kg})$ in $50 \%$ ethanol, $10 \%$ HCO-60 and $10 \%$ propylene glycol was injected via the femoral vein. Blood samples (350 $\mu \mathrm{l}$ each) were collected at designated time intervals from the jugular vein under light ether anesthesia. Plasma was separated by centrifugation at $3000 \times \boldsymbol{g}$ for $10 \mathrm{~min}$ and stored at $-30^{\circ} \mathrm{C}$. The HPLC assays for caffeine, ${ }^{17)}$ chlorzoxazone, ${ }^{18,19)}$ doxorubicin ${ }^{20)}$ lidocaine, ${ }^{21)}$ tolbutamide ${ }^{22)}$ and zonisamide ${ }^{23)}$ were carried out according to the cited methods. The blood concentration of cyclosporin A or tacrolimus was measured with a TDx or an IMx analyzer using a commercial assay kit (Tacrolimus II, Dainabot Co., Ltd., Tokyo, Japan), according to the manufacturer's instructions.

Data Analysis The pharmacokinetic parameters were estimated according to model-independent moment analysis as described by Yamaoka et $a{ }^{24)}$ The data were analyzed using Student's $t$ test to compare the unpaired mean values of two
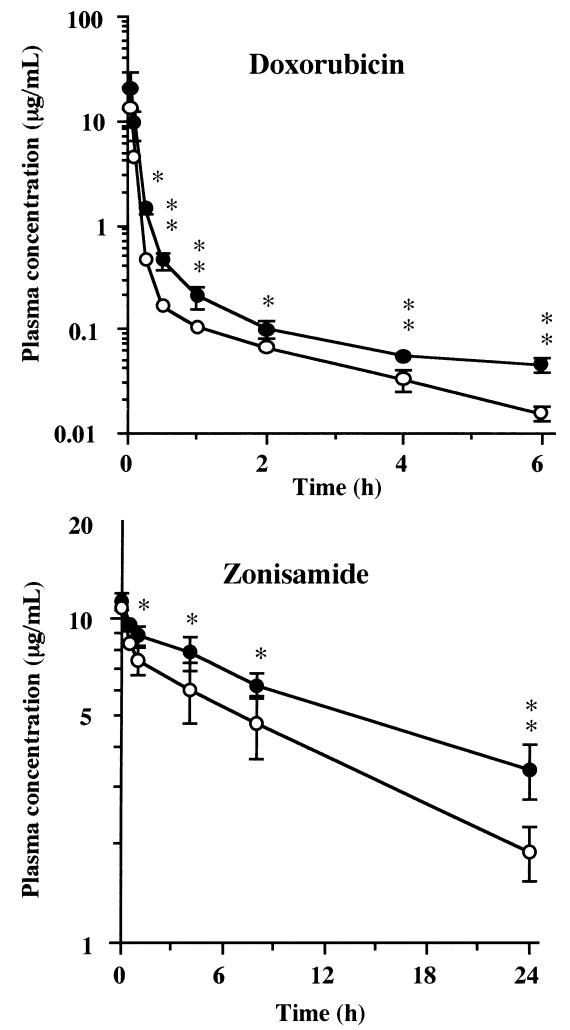

Fig. 1. Blood or Plasma Concentration-Time Courses of Doxorubicin, Tacrolimus, Cyclosporin A and Zonisamide after Intravenous Administration to Vehicle Control $(\mathrm{O})$ and $\mathrm{CCl}_{4}$-Treated $(\bigcirc)$ Rats

Rats were orally treated with $\mathrm{CCl}_{4}(0.5 \mathrm{ml} / \mathrm{kg})$ at $24 \mathrm{~h}$ prior to doxorubicin $(5 \mathrm{mg} / \mathrm{kg})$, tacrolimus $(2 \mathrm{mg} / \mathrm{kg})$, cyclosporin A (10 mg/kg) or zonisamide (10 mg/kg) administration. Each point with a bar represents the mean \pm S.D. of four rats. 
Table 1. Pharmacokinetic Parameters of Various Drugs after i.v. Administration in Untreated and $\mathrm{CCl}_{4}$-Treated $\mathrm{Rats}$

\begin{tabular}{|c|c|c|c|c|c|c|c|c|}
\hline \multirow{2}{*}{ Parameters } & \multicolumn{2}{|c|}{ Cyclosporin A } & \multicolumn{2}{|c|}{ Doxorubicin } & \multicolumn{2}{|c|}{ Tacrolimus } & \multicolumn{2}{|c|}{ Zonisamide } \\
\hline & No treatment & $\mathrm{CCl}_{4}$ treatment $^{a)}$ & No treatment & $\mathrm{CCl}_{4}$ treatment $^{a)}$ & No treatment & $\mathrm{CCl}_{4}$ treatment $^{a)}$ & No treatment & $\mathrm{CCl}_{4}$ treatment $^{a}$ \\
\hline$A U C(\mathrm{mg} \mathrm{h} / \mathrm{ml})$ & $21.7 \pm 1.8$ & $73.2 \pm 7 * *$ & $1.61 \pm 0.05$ & $3.17 \pm 0.34 * *$ & $857 \pm 61$ & $2060 \pm 190 * *$ & $133 \pm 12$ & $215 \pm 19 * *$ \\
\hline$M R T(\mathrm{~h})$ & $4.08 \pm 0.52$ & $9.25 \pm 1.51 * *$ & $0.671 \pm 0.05$ & $1.19 \pm 0.09 * *$ & $1.81 \pm 0.20$ & $4.43 \pm 0.71 * *$ & $17.2 \pm 2.4$ & $23.5 \pm 3.7^{*}$ \\
\hline$V d_{\mathrm{ss}}(\mathrm{ml} / \mathrm{kg})$ & $1880 \pm 310$ & $1260 \pm 225^{*}$ & $2080 \pm 160$ & $1870 \pm 250$ & $4210 \pm 630$ & $4290 \pm 870$ & $1300 \pm 250$ & $1090 \pm 230$ \\
\hline$C L_{\text {tot }}(\mathrm{ml} / \mathrm{h} / \mathrm{kg})$ & $462 \pm 33$ & $137 \pm 9 * *$ & $3100 \pm 80$ & $1580 \pm 160 * *$ & $2330 \pm 150$ & $968 \pm 80 * *$ & $75.4 \pm 6.4$ & $46.5 \pm 3.9^{* *}$ \\
\hline$T_{1 / 2}(\mathrm{~h})$ & $3.47 \pm 0.31$ & $6.83 \pm 1.1^{* *}$ & $1.81 \pm 0.13$ & $3.50 \pm 0.28 * *$ & $1.56 \pm 0.22$ & $3.39 \pm 0.42 * *$ & $12.1 \pm 1.8$ & $16.2 \pm 2.5^{*}$ \\
\hline$A S T(\mathrm{IU} / \mathrm{l})$ & $88 \pm 8$ & $2880 \pm 360^{* *}$ & $88 \pm 7$ & $1340 \pm 390 * *$ & $99 \pm 9$ & $1960 \pm 250 * *$ & $83 \pm 5$ & $3940 \pm 850 * *$ \\
\hline
\end{tabular}

Each value represents the mean \pm S.D. of four rats. $* * *$ Significantly different from the untreated rats at $p<0.05$ and 0.01, respectively. $a$ ) Dose: $0.5 \mathrm{ml} / \mathrm{kg} \mathrm{CCl} \mathrm{l}_{4}$

sets of data. The number of determinations is noted in each table and figure. A value of $p<0.05$ or 0.01 was taken to indicate a significant difference between sets of data.

\section{RESULTS}

Blood or Plasma Concentration-Time Courses of Various Drugs Untreated control rats and $\mathrm{CCl}_{4}$-treated rats were intravenously injected with cyclosporin A $(10 \mathrm{mg} / \mathrm{kg})$, doxorubicin $(5 \mathrm{mg} / \mathrm{kg})$, tacrolimus $(2 \mathrm{mg} / \mathrm{kg})$ or zonisamide $(10 \mathrm{mg} / \mathrm{kg})$. As shown in Table 1 , the $A S T$ values of the $\mathrm{CCl}_{4}$ treated groups were about $15-50$ times higher than those of the untreated control groups. The blood (cyclosporin A, tacrolimus) or plasma (doxorubicin, zonisamide) concentrations of these four drugs in the $\mathrm{CCl}_{4}$-treated rats were all significantly higher than those in the control rats (Fig. 1). The pharmacokinetic parameters of these drugs are listed in Table 1. Each value of the area under the blood or plasma concentration-time curve from 0 time to infinity $(A U C)$ of the $\mathrm{CCl}_{4^{-}}$ treated group was significantly larger than that of the control group. The values of $C L_{\text {tot }}$ of these drugs in the $\mathrm{CCl}_{4}$-treated rats were decreased to about $1 / 2-1 / 3$ of those in control rats, whereas the values of distribution volume at the steady-state $\left(V d_{\mathrm{ss}}\right)$ were not significantly different between the two groups. The $V d_{\text {ss }}$ value for cyclosporin $\mathrm{A}$ in the $\mathrm{CCl}_{4}$-treated rats became significantly less than that in the control rats, but the change was small.

Correlation between $\boldsymbol{A S T}$ and Hepatic Intrinsic Clearance The pharmacokinetic parameters for cyclosporin A, doxorubicin, tacrolimus, and zonisamide in this study (Table 1) and those for caffeine $(5 \mathrm{mg} / \mathrm{kg})$, chlorzoxazone $(10$ $\mathrm{mg} / \mathrm{kg})$, lidocaine $(10 \mathrm{mg} / \mathrm{kg})$, and tolbutamide $(10 \mathrm{mg} / \mathrm{kg})$ obtained in previous study ${ }^{13}$ were used for the analyses. The $C L_{\text {tot }}$ values were calculated by model-independent moment analysis from the blood or plasma concentrationtime courses of these drugs. Further, the hepatic intrinsic clearance $\left(C L_{\text {int }}\right)$ was calculated by using Eq. 1:

$$
C L_{\text {tot }}^{\mathrm{P}}=R B P \cdot C L_{\text {tot }}^{\mathrm{B}}=\frac{Q_{\mathrm{h}} \cdot f_{\mathrm{p}} \cdot C L_{\text {int }}}{Q_{\mathrm{h}}+f_{\mathrm{p}} \cdot C L_{\text {int }} / R B P}
$$

where $C L_{\text {tot }}^{\mathrm{P}}$ and $C L_{\text {tot }}^{\mathrm{B}}$ represent the total clearances of plasma and blood, respectively. $Q_{\mathrm{h}}, R B P$ and $f_{\mathrm{p}}$ represent the hepatic blood flow, the blood-to-plasma partition coefficient and the plasma unbound fraction, respectively. The values of $Q_{\mathrm{h}}$ were measured using indocyanine green in rats at $24 \mathrm{~h}$ after treatment with $\mathrm{CCl}_{4}(0.01-0.5 \mathrm{ml} / \mathrm{kg})$. The $Q_{\mathrm{h}}$ value in six normal rats was $58 \pm 7 \mathrm{ml} / \mathrm{min} / \mathrm{kg}$ (mean \pm S.D.), whereas that in rats with $\mathrm{CCl}_{4}$ treatment did not differ significantly from that
Table 2. Pharmacokinetic Parameters in Normal Rats

\begin{tabular}{lcccc}
\hline \hline & $f_{\mathrm{p}}^{a)}$ & $R B P^{b)}$ & $C L_{\mathrm{int, \textrm {N }}}{ }^{c)}$ & slope $^{d)}$ \\
\hline Caffeine & $0.8^{e)}$ & $1.15 \pm 0.02$ & 9.19 & $5.11 \times 10^{-4}$ \\
Chlorzoxazone & $0.026 \pm 0.013^{f)}$ & $1.22 \pm 0.02$ & 117 & $1.02 \times 10^{-4}$ \\
Cyclosporin A & $0.05^{g)}$ & $1.21 \pm 0.08$ & 215 & $1.65 \times 10^{-4}$ \\
Doxorubicin & $0.344^{h)}$ & $2.15 \pm 0.13$ & 257 & $2.54 \times 10^{-4}$ \\
Lidocaine & $0.38^{i)}$ & $0.99 \pm 0.11$ & 7596 & $6.02 \times 10^{-4}$ \\
Tacrolimus & $0.1^{g)}$ & $1.28 \pm 0.12$ & 1500 & $3.31 \times 10^{-4}$ \\
Tolbutamide & $0.045^{j)}$ & $0.76 \pm 0.03$ & 7.12 & $2.90 \times 10^{-5}$ \\
Zonisamide & $0.514^{k)}$ & $0.84 \pm 0.14$ & 2.52 & $4.95 \times 10^{-5}$ \\
\hline
\end{tabular}

a) Unbound fraction in plasma. b) Blood to plasma concentration ratio (mean \pm S.D., $n=3$ ). c) Hepatic intrinsic clearance in normal rats was calculated from the total clearance shown in Table 1 and the data of Yokogawa et al. ${ }^{13)}(\mathrm{ml} / \mathrm{min} / \mathrm{kg})$. d) Slope of the linear regression equation for each drug shown in Figs. 3 and 4. e) Ref. 41, f) determined value (mean \pm S.D., $n=3$ ), $g$ ) ref. $42, h$ ) ref. $43, i$ ) ref. $21, j$ ) ref. $22, k$ ) ref. 44.

in normal rats. The $R B P$ and $f_{\mathrm{p}}$ value of each drug are shown in Table 2. Further, rats with $\mathrm{CCl}_{4}$ treatment $(0.01-$ $0.5 \mathrm{ml} / \mathrm{kg}$ ) were intravenously injected with the eight drugs, and then the $C L_{\text {tot }}$ values for these drugs were calculated by model-independent moment analysis. The $C L_{\text {int }}$ values were estimated by substituting the values of $C L_{\mathrm{tot}}, Q_{\mathrm{h}}, f_{\mathrm{p}}$ and $R B P$ into Eq. 1. Figure 2 shows the correlations between $A S T$ and $C L_{\text {int }}$ for the eight drugs. The linear regression equation is represented as follows (Eq. 2):

$$
C L_{\text {int }}=b \cdot 10^{(- \text {slope } A S T)}
$$

where $b$ and slope are the intercept of the ordinate and the slope of the linear regression equation, respectively. Good correlations were observed between them for the eight drugs $(r=0.733-0.949)$, but the slopes showed clear differences among the eight drugs (Table 2).

Correlation between the Slope and Hepatic Intrinsic Clearance of Normal Rats The $C L_{\text {tot }}$ values were converted to hepatic intrinsic clearance of normal rats $\left(C L_{\text {int, } N}\right)$ by using Eq. 1 . These values of $C L_{\text {int,N }}$ are shown in Table 2. Figure 3 shows the correlation between the slope and the $C L_{\text {int, N }}$ value for the eight drugs. A good correlation was found for seven drugs, excluding caffeine $(r=0.953)$. The following linear regression equation (Eq. 3) was obtained.

$$
\text { slope }=0.0000226 \cdot C L_{\mathrm{int}, \mathrm{N}}^{0.381}
$$

\section{DISCUSSION}

Here, we propose a novel method which allows us to predict the decrease of $C L_{\text {tot }}$ during acute hepatic failure, based on the serum $A S T$ activity. 

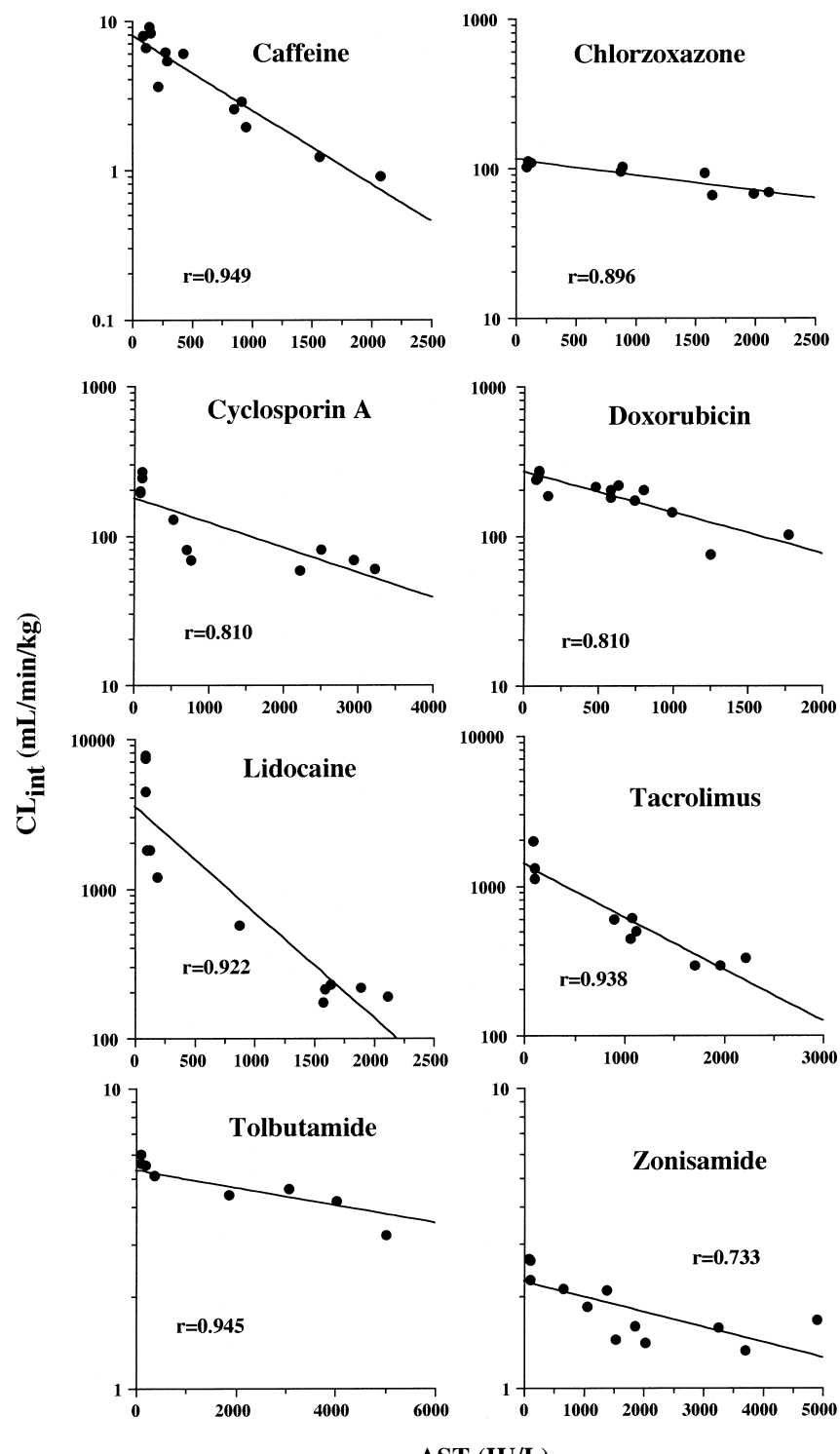

AST (IU/L)

Fig. 2. Correlation between the Serum $A S T$ and $C L_{\text {int }}$ of Each Drug after Intravenous Administration to Vehicle Control and $\mathrm{CCl}_{4}$-Treated Rats

Rats were orally treated with $\mathrm{CCl}_{4}(0.01-0.5 \mathrm{ml} / \mathrm{kg})$ at $24 \mathrm{~h}$ prior to doxorubicin $(5 \mathrm{mg} / \mathrm{kg})$, chlorzoxazone $(10 \mathrm{mg} / \mathrm{kg})$, caffeine $(5 \mathrm{mg} / \mathrm{kg})$, cyclosporin A $(10 \mathrm{mg} / \mathrm{kg})$, lidocaine $(10 \mathrm{mg} / \mathrm{kg})$, tacrolimus $(2 \mathrm{mg} / \mathrm{kg})$, tolbutamide $(10 \mathrm{mg} / \mathrm{kg})$ or zonisamide $(10 \mathrm{mg} / \mathrm{kg})$ administration

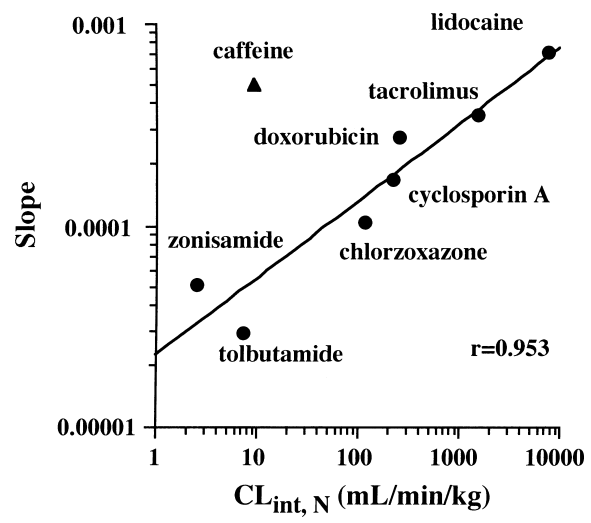

Fig. 3. Correlation between the Slope and the Hepatic Intrinsic Clearance of Normal Rat $\left(C L_{\text {int,N }}\right)$

The slope value is obtained from the linear regression equation for each drug as shown in Fig. 3.
We prepared model rats with various degrees of acute hepatic failure induced by administering various doses of $\mathrm{CCl}_{4}$. To examine the degree of $C L_{\text {tot }}$ decrease in acute hepatic failure, we selected eight probe drugs which are metabolized by various CYP isoforms: caffeine ${ }^{25}$ by CYP1A2, doxorubicin $^{26)}$ by CYP2B1, tolbutamide ${ }^{27)}$ by CYP2C11, chlorzoxazone $^{28)}$ by CYP2E1, and cyclosporine $\mathrm{A},{ }^{29)}$ lidocaine, ${ }^{30}$ tacrolimus $^{29)}$ and zonisamide ${ }^{31)}$ by CYP3A2. We found that the blood or plasma concentrations of these drugs in $\mathrm{CCl}_{4}$ treated rats were significantly higher than those in control rats (Fig. 1). The $A U C$ values in $\mathrm{CCl}_{4}$-treated rats were significantly larger than those in control rats for all the drugs (Table 1). The values of $C L_{\text {tot }}$ in the $\mathrm{CCl}_{4}$-treated rats were significantly smaller than those in control rats, whereas the values of $V d_{\mathrm{ss}}$ were not significantly different. However, we found that the ratio of decrease of $C L_{\text {tot }}$ in the $\mathrm{CCl}_{4}$-treated group versus the control group ranged from 30 to $60 \%$ among these drugs.

There are two main factors affecting the hepatic clearance, i.e., hepatic blood flow and drug-metabolizing activity. In general, the $Q_{\mathrm{h}}$ is increased in patients with acute liver disease, ${ }^{32)}$ and unchanged in patients with acute viral hepatitis, ${ }^{33)}$ but decreased in patients with chronic liver disease. ${ }^{34,35)}$ It has been reported that the $Q_{\mathrm{h}}$ in rats with acute hepatic failure is increased by $\mathrm{CCl}_{4}$ treatment, ${ }^{36,37)}$ but in this study we could not confirm the increase of $Q_{\mathrm{h}}$ in rats with $\mathrm{CCl}_{4}$ treatment compared with normal rats. Further, we considered that the values of $f_{\mathrm{p}}$ and $R B P$ in rats with acute hepatic failure did not differ from those in normal rats, because there was no significant difference in serum albumin concentration between the two groups. ${ }^{13)}$ It is well known that basic drugs generally bind to $\alpha_{1}$-acid glycoprotein (AGP). It is reported that serum AGP concentration increases in acute cholecystitis $^{38)}$ and decreases in cirrhosis. ${ }^{39)}$ Therefore, the AGP concentration might be altered in rats with acute hepatic failure. However, we considered that the influence of a change in binding to AGP was likely to be negligible, because most basic drugs, such as lidocaine, show hepatic blood flow-limited clearance. Thus, the obtained values of $C L_{\text {tot }}$ for these drugs were used to calculate the values of $C L_{\text {int }}$ according to Eq. 1, taking into account the values of $Q_{\mathrm{h}}(58 \mathrm{ml} / \mathrm{min} / \mathrm{kg}), f_{\mathrm{p}}$ and $R B P$ (Table 2). There was a good correlation between $C L_{\text {int }}$ and $A S T$ activity for each drug (Fig. 2). The correlation with $A S T$ was the highest among seven laboratory data in our previous report (data not shown). ${ }^{13)}$

The results indicate that it may be possible to predict the decrease of $C L_{\text {tot }}$ from laboratory data on $A S T$. However, we found that the ratios of decrease of $C L_{\mathrm{int}}$ against an increase in $A S T$ (slope of the plots) vary markedly among the eight drugs. We previously reported that there is a good negative correlation between serum $A S T$ activity and the hepatic enzyme activity of each CYP in the liver of rats with acute hepatic failure, but the decreasing ratio of hepatic enzyme activity differs among CYP isoforms. ${ }^{13)}$ We have found that there is no agreement between the decreasing ratio of $C L_{\text {int }}$ of eight drugs with an increase in $A S T$ value (in vivo) and the decreasing ratio of CYP isoform activity for each drug (in vitro $){ }^{13)}$ One reason for this may be the difference between the probe drugs used in in vivo studies and the substrates used for measurement of the activity of CYP isoforms. Further, the decreasing ratio of $C L_{\text {tot }}$ with an increase in $A S T$ dif- 
fers among the probe drugs metabolized by the same kind of CYP isoform. This may be because the in vivo results are dependent on various characteristics of the drug, such as lipophilicity, affinity for the CYP isoform, change of $f_{\mathrm{p}}$, and induction of drug transporter(s) by $\mathrm{CCl}_{4}$ treatment. Therefore, it does not seem feasible to predict the decreasing ratio of $C L_{\text {tot }}$ from the results of in vitro studies.

However, these results indicate that determination of the correlation for each drug may be necessary to predict its $C L_{\text {tot }}$ value from the $A S T$ value. Thus, we examined whether the slopes of these drugs shown in Fig. 2 are available for the prediction of $C L_{\text {tot }}$ for unknown drugs in acute hepatic failure. There was not a good correlation between the slope and CYP isoform or lipophilicity among these drugs. However, we found a good correlation between the slopes and $C L_{\text {int,N }}$ values for seven drugs, excluding caffeine (Fig. 3). Caffeine probably deviated from the correlation line because it is a nonlinearly metabolized drug. ${ }^{40)}$ However, we could not explain why the greater the $C L_{\text {int, N }}$ value, the greater the degree of decreasing ratio of $C L_{\text {int }}$ against an increase of $A S T$.

Then, we tried to predict the degree of decrease of $C L_{\text {tot }}$ of a model drug from the serum $A S T$ value in rats with acute hepatic failure. The slope for a model drug can be predicted by substituting the $C L_{\text {int,N }}$ value of this drug into Eq. 3 . When the obtained slope and normal $A S T$ value (85 IU/1) are substituted into Eq. 2, the $b$ value of this drug can be estimated. Thus, we believe that the $C L_{\text {int }}$ of a model drug in acute hepatic failure can be predicted by substituting the $A S T$ value into Eq. 2 using the obtained values of the slope and $b$ value for the drug.

In conclusion, we have established a novel method for predicting the ratio of decrease of $C L_{\text {tot }}$ in rats with acute hepatic failure from the $A S T$ value. The validity of scale-up from rats to humans with acute hepatic failure will need to be confirmed, but this method may be useful for estimating dose modification of drugs in the clinic.

\section{REFERENCES}

1) Kerremans A. L., Neth. J. Med., 48, 237-243 (1996).

2) Guengerich F. P., Turvy C. G., J. Pharmacol. Exp. Ther, 256, 1189 1894 (1991).

3) Bowen W. P., Carey J. E., Miah A., McMurray H. F., Munday P. W., James R. S., Coleman R. A., Brown A. M., Drug Metab. Dispos., 28, $781-788(2000)$

4) Rodriguez-Antona C., Donato M. T., Pareja E., Gomez-Lechon M. J., Castell J. V., Arch. Biochem. Biophys., 393, 308-315 (2001).

5) Finnstrom N., Thorn M., Loof L., Rane A., Eur. J. Clin. Pharmacol., 57, 403-409 (2001).

6) Panduro A., Valencia J., Rojkind M., Int. J. Biochem., 25, 525-532 (1993).

7) Kimura T., Nakayama S., Yamao T., Kurosaki Y., Nakayama T., Biol. Pharm. Bull., 16, 1140-1145 (1993).

8) Kleinbloesem C. H., van Harten J., Wilson J. P., Danhof M., van Brummelen P., Breimer D. D., Clin. Pharmacol. Ther, 40, 21-28 (1986).

9) Frye R. F., Matzke G. R., Adedoyin A., Porter J. A., Branch R. A., Clin. Pharmacol. Ther, 62, 365-376 (1997).

10) Tucker G. T., Rostami-Hodjegan A., Jackson P. R., Xenobiotica, 28, 1255-1273 (1998).

11) Krecic-Shepard M. E., Barnas C. R., Slimko J., Gorski J. C., Wainer I W., Schwartz J. B., Clin. Pharmacol. Ther., 66, 40-50 (1999).

12) Matsuo K., Kosugi T., Kusano A., Obayashi M., Kurokawa M., Jpn. J.
$T D M, 20,42-47$ (2003).

13) Yokogawa K., Watanabe M., Takeshita H., Nomura M., Mano Y., Miyamoto K., Int. J. Pharm., 269, 479-489 (2004).

14) Perez Tamayo R., Hepatology, 3, 112-120 (1983).

15) Munoz Torres E., Paz Bouza J. I., Abad Hernandez M. M., Alonso Martin M. J., Lopez Bravo A., Int. J. Tissue React., 10, 245-251 (1988).

16) Yokota M., Iga T., Awazu S., Hanano M., J. Appl. Physiol., 41, 439441 (1976).

17) Kawahara M., Kagiyama H., Kanazawa Y., Tsuchiya H., Tomita K., Yokogawa K., Miyamoto K., Biopharm. Drug Dispos., 25, 61-67 (2004).

18) Chen L., Yang C. S., Life Sci., 58, 1575-1585 (1996).

19) Mizuno D., Tanaka E., Tanno K., Misawa S., Arch. Toxicol., 74, 139144 (2000).

20) Shinozawa S., Oda T., J. Chromatogr., 212, 323-330 (1981).

21) Shibasaki S., Kawamata Y., Ueno F., Koyama C., Itho H., Nishigaki R., Uemura K., J. Pharmacobio-Dyn., 11, 785-793 (1988).

22) Matunaga N., Nishijima T., Hattori K., Iizasa H., Yamamoto K., Kizu J., Takanaka A., Morikawa A., Nakashima E., Biol. Pharm. Bull., 24, 1305-1310 (2001).

23) Nakamura M., Hirade K., Sugiyama T., Katagiri Y., J. Chromatogr. B, Biomed. Sci., Appl., 755, 337-341 (2001).

24) Yamaoka K., Nakagawa T., Uno T., J. Pharmacokinet. Biopharm., 6, $547-558$ (1978)

25) Matsunaga N. K., Isawa M., Kizu J., Miyazaki N., Takanaka A., Nakashima E., Drug Metab. Pharmacokinet., 18, 296-302 (2003).

26) Ellouk-Achard S., Martin C., Pham-Huy C., Duc H. T., Thevenin M., Dutertre-Catella H., Warnet J. M., Claude J. R., Arch. Toxicol., 71, 437-442 (1997).

27) Goeptar A. R., Te Koppele J. M., Lamme E. K., Pique J. M., Vermeulen N. P., Mol. Pharmacol., 44, 1267-1277 (1993).

28) Matsunaga N., Nishijima T., Hattori K., Iizasa H., Yamamoto K., Kizu J., Takanaka A., Morikawa A., Nakashima E., Biol. Pharm. Bull., 24, 1305-1310 (2001).

29) Kim Y. G., Cho M. K., Kwon J. W., Kim S. G., Chung S. J., Shim C. K., Le Myung G., Biopharm. Drug Dispos., 23, 121-129 (2002).

30) Huang Y. S., Lee S. D., Deng J. F., Wu J. C., Lu R. H., Lin Y. F., Wang Y. J., Lo K. J., J. Hepatol., 19, 140-147 (1993).

31) Nakasa H., Komiya M., Ohmori S., Rikihisa T., Kitada M., Drug Metab. Dispos., 21, 777-781 (1993).

32) Clemmesen O., Dan. Med. Bull., 49, 177-193 (2002).

33) Preisig R., Morris T. Q., Shaver J. C., Christy N. P., J. Clin. Invest., 45, 1379-1387 (1966).

34) Branch R. A., Morgan M. H., James J., Read A. E., Gut, 17, 975-983 (1976).

35) Taniguchi H., Oguro A., Koyama H., Masuyama M., Takahashi T., J. Comput. Assist. Tomogr., 20, 135-144 (1996).

36) Kurihara T., Kitamura Y., Adachi Y., Obuchi M., Abe K., Akimoto M., Hashimoto H., Ishiguro H., Niimi A., Maeda A., Shigemoto M., Yamashita K., Yokoyama I., J. Gastroenterol. Hepatol., 11, 978-984 (1996).

37) Tanaka N., Tanaka K., Nagashima Y., Kondo M., Sekihara H., Gastroenterology, 117, 173-180 (1999).

38) Adamian A. I., Guliaev A. A., Ivanina T. A., Evteeva E. A., Samsonov V. T., Klin. Lab. Diagn., 1997, 8-10 (1997).

39) Belpaire F. M., Bogaert M. G., Prog. Clin. Biol. Res., 300, 337-350 (1989).

40) Bortolotti A., Jiritano L., Bonati M., Drug Metab. Dispos., 13, 227 231 (1985).

41) Bonati M., Garattini S., "Caffeine-Perspectives from Recent Research," ed. by Dews P. B., Springer-Verlag, Berlin, 1984, pp. 48-56.

42) Takada K., Usuda H., Oh-Hashi M., Biopharm. Drug Dispos., 13, $345-355$ (1992).

43) Terasaki T., Iga T, Sugiyama Y., Hanano M., J. Pharm. Sci., 73, 1359-1363 (1984).

44) Ito T., Yamaguchi T., Miyazaki H., Sekine Y., Shimizu M., Ishida S., Yagi K., Kakegawa N., Seino M., Wada T., Arzneim.-Forsch., 32, $1581-1586$ (1982). 